

\title{
Enhancement in the field sensitivity of magnetoelectric laminate heterostructures
}

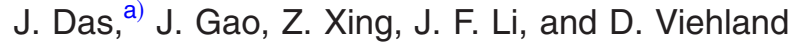 \\ Department of Materials Science and Engineering, Virginia Tech, Blacksburg, Virginia 24061, USA
}

(Received 24 June 2009; accepted 16 August 2009; published online 2 September 2009)

\begin{abstract}
The effect of magnetostrictive layer thickness on the magnetoelectric (ME) response and field sensitivity of $\mathrm{Pb}(\mathrm{Zr}, \mathrm{Ti}) \mathrm{O}_{3}$-metglas based sandwiched $\mathrm{ME}$ heterostructures has been studied. Such structures hold promise for sensor applications. The increase in metglas thickness results in a significant increase in the ME response and magnetic field sensitivity. The ME coefficient and field sensitivity increase by about $1.5-1.75$ and 2.7 times, respectively, for a structure with $150 \mu \mathrm{m}$ thick six metglas layers on both sides of the $\mathrm{Pb}(\mathrm{Zr}, \mathrm{Ti}) \mathrm{O}_{3}$, in comparison to a $50 \mu \mathrm{m}$ thick two layered structure. (C) 2009 American Institute of Physics. [DOI: 10.1063/1.3222914]
\end{abstract}

Engineered magnetoelectric (ME) heterostructures have been investigated due to the potential to control the electric/magnetic response with magnetic/electric fields. ${ }^{1-4}$ Most of these structures, reported so far, have generally been comprised of two phases: one magnetostrictive phase and one piezoelectric phase, in either layered ${ }^{5}$ or nanopillar-embedded-in-matrix ${ }^{6}$ form. Neither of these two phases individually exhibit magnetoelectricity. However, in the heterostructure form where the two phases are engineered to stay in close contact, the two different ferroic degrees of freedom interact with each other, producing a remarkable control of the electric response with magnetic fields or vice versa. The achievement of such control in the ME heterostructures promises multifunctional sensor, memory, and communication devices with low cost, small size, and low power budgets.

The option to control the electric response with magnetic fields opens up the possibility to use engineered ME structures as magnetic field sensors. Such laminate layered structures with strong ME couplings (represented by ME voltage coefficient $\alpha_{V}$ in the range of a few $\mathrm{V} / \mathrm{cm}-\mathrm{Oe}$ ) enables one to detect magnetic fields in the nanoTesla $(\mathrm{nT})$ range at room temperature with a simple operational amplifer. ${ }^{7}$ Different combinations of relative directions of the magnetization and polarization of various piezoelectric [for example, $\left.\mathrm{Pb}(\mathrm{Zr}, \mathrm{Ti}) \mathrm{O}_{3}(\mathrm{PZT})\right]$ and magnetostrictive phases (for example, metglas) have been attempted so far. ${ }^{8-10}$ For example, the push-pull mode is a combination that consists of a structure with a longitudinally magnetized magnetic layer and a symmetrically poled piezoelectric layer about its central nodal point in reverse directions along the longitudinal axis. It has been found that three layer metglas-PZT-metglas structures offer the highest ME voltage coefficient and magnetic field sensitivity. ${ }^{11}$

In order to compete with magnetic field sensors presently available, there has been a critical need to further increase the ME coupling and field sensitivity of the metglasPZT-metglas structures. Application of the magnetic field in these heterostructures changes the magnetostriction of the metglas layers that results in a strain on the adjacent PZT fibers. This strain affects the polarization of the piezoelectric

${ }^{a)}$ Electronic mail: jaydip@vt.edu.
PZT fibers and thus, it offers the potential to tune the electric response with applied magnetic fields. The amount of strain on the PZT fibers from the metglas depends on the thickness ratios of the metglas and PZT layers. One can optimize the metglas-PZT thickness ratio to obtain an improved ME response for the structure. Recent theoretical studies have also indicated a strong dependence of the ME coefficient on the thickness ratios of the magnetic and electric layers. ${ }^{12}$ However, there has been no experimental report so far on the dependence of the ME coefficient and field sensitivity on thickness ratios for the metglas-PZT laminate structures.

This letter reports the achievement of a significantly higher ME coefficient and magnetic field sensitivity for an optimized metglas-PZT thickness ratio. The dimensions of the metglas-PZT-metglas sandwiched structure have also been optimized to make it more practical for device applications. The ME coefficients show an increase of about 1.51.75 times from the previously reported metglas-PZT thickness ratio, ${ }^{11}$ resulting in a 2.7 times enhancement in the field sensitivity of the ME sensors.

Thin polymer insulating films with interdigitated (ID) copper electrodes were attached to both top and bottom of five $200 \mu \mathrm{m}$ thick commercial PZT fibers (CTS Wireless, Albuquerque, NM) with epoxy resin to obtain the push-pull geometry. The dimension of each PZT fiber was 0.2 $\times 4.2 \mathrm{~cm}^{2}$ and the distance between the electrodes was $850 \mu \mathrm{m}$. The $25 \mu \mathrm{m}$ thick Metglas foils (Metglas Inc., Conway, SC) with a saturation magnetostriction of about 27 $\mathrm{ppm}$, were cut to the widths of about $1 \mathrm{~cm}$ to match the total width of the five PZT fibers. The choice of the metglas length of $7.5 \mathrm{~cm}$ was based on the trade-off between the need of about $10 \mathrm{~cm}$ long metglas for maximum magnetic flux concentration $^{11}$ and the necessity to reduce the device size for practical applications. The cut metglas pieces were then stacked one on top of each other, bonded with epoxy resin, and were pressed using a hydraulic press to minimize the epoxy thickness in-between the metglas foils. Two samples each of 2, 4, 6, 8, and 10 metglas stacks were made. Metglas stacks with equal number of layers were then attached at the top and bottom of the ID electrode-PZT-ID electrode structure with epoxy, in order to obtain the ME laminate layered structures.

The voltages induced across the ID electrodes of the PZT fibers were then measured with a lock-in amplifier as a 
(a)
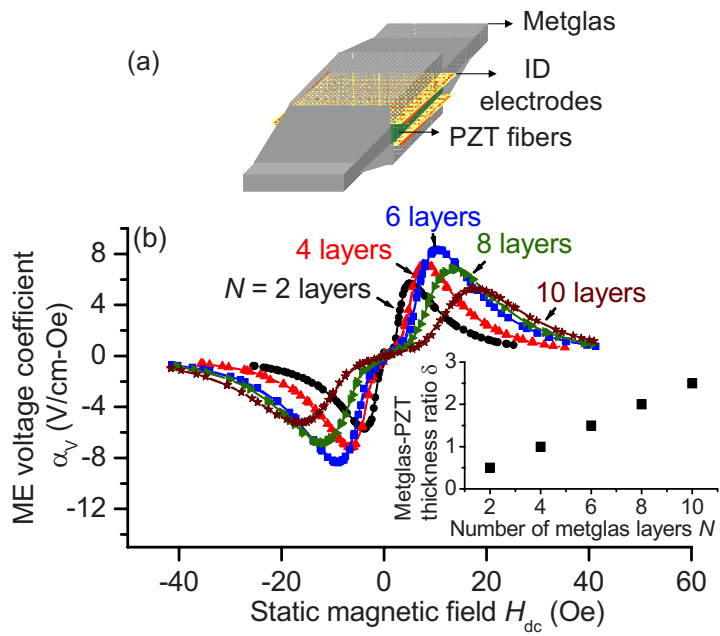

FIG. 1. (Color online) (a) Schematic diagram of the PZT-metglas structure. (b) ME voltage coefficient $a_{V}$ as a function of the static dc magnetic field $H_{\mathrm{dc}}$ for various PZT fiber-metglas laminate composites. The numbers indicate the number of metglas layers $(N)$ on both side of the PZT fiber. The inset shows the metglas-PZT thickness ratio $\delta$ as a function of $N$.

function of the dc magnetic field $H_{\mathrm{dc}}$ and in response to a Helmholtz coil driven ac field of $H_{\mathrm{ac}}=1$ Oe at a frequency $f=1 \mathrm{kHz}$. Both dc and ac fields were applied along the length of the heterostructure. Figure 1 shows (a) a schematic diagram of a metglas-PZT heterostructure with components as indicated and (b) the ME voltage coefficient $\alpha_{V}$ as a function of $H_{\mathrm{dc}}$ for different number of metglas layers $(N)$ stacked on both sides of PZT fibers. From this figure, it can be seen that $\alpha_{V}$ is nearly zero at $H_{\mathrm{dc}}=0$; increases as $H_{\mathrm{dc}}$ is increased; reaches a maximum at a particular bias field; and subsequently decreases as $H_{\mathrm{dc}}$ is further increased. One can also clearly see that the maximum value of $\alpha_{V}$ increases with increasing number of metglas layers until $N=6$, after which it begins to decrease with further increase in $N$. Moreover, the $H_{\mathrm{dc}}$ required for the maximum $\alpha_{V}$ response also increases with $N$. The inset in (b) shows the metglas-PZT thickness ratio $\delta$ as a function of $N$, where one can see the expected linear increase in $\delta$ with $N$.

Figure 2 shows a summary of the data given in Fig. 1. This figure also provides additional data for the output charge across the ID electrodes for the structures with different layered stacks, and a comparison with the theoretical predictions. The charge response was measured with a lock-in amplifier and a charge preamplifier as a function of $H_{\mathrm{dc}}$ for a $H_{\mathrm{ac}}=1$ Oe at $f=1 \mathrm{kHz}$. Parts (a) and (b) show the maximum ME voltage and charge coefficients $\left(\alpha_{V \text {-MAX }}\right.$ and $\alpha_{C \text {-MAX }}$, respectively) as a function of $N$. Arrows indicate the points where the ME coefficients are maximum. One sees that $\alpha_{V \text {-MAX }}$ and $\alpha_{C \text {-MAX }}$ both increase with increasing $N$ up to $N=6$, and then subsequently decrease for $N>6$. The 1.5 and 1.75 times increase in the $\alpha_{V \text {-MAX }}$ and $\alpha_{C \text {-MAX }}$ values, respectively, for $N=6$ relative to $N=2$ is noteworthy. It should also be noted that all prior reports have focused on $N=2$. Part (c) shows that the $H_{\mathrm{dc}}$ required to achieve the maximum ME response increases linearly with $N$. Part (d) shows the normalized ME voltage coefficient as a function of the thickness ratio $\delta$ for the present study and theoretical predictions from Ref. 12 for $\mathrm{NiFe}_{2} \mathrm{O}_{4}$-PZT structure, as indicated. One can see that, in both the cases, the overall trend in the ME coefficient as a function of $\delta$ looks nearly similar.
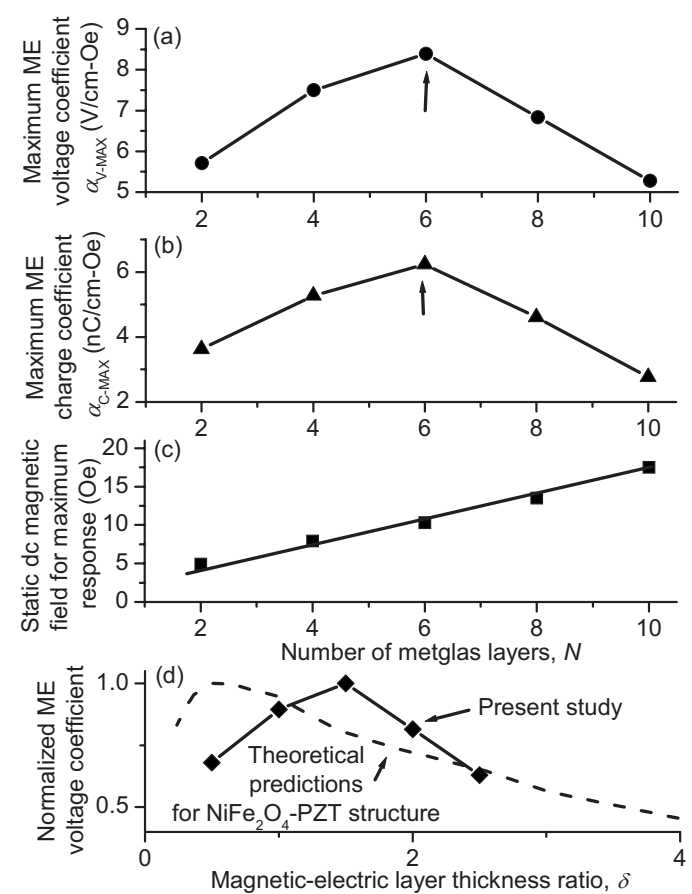

FIG. 2. Maximum ME (a) voltage $\left(\alpha_{V \text {-MAX }}\right)$ and (b) charge $\left(\alpha_{C \text {-MAX }}\right)$ coefficients as a function of the number of metglas layers $N$ on both sides of the PZT layer. The points show the data and the lines are drawn as a guide to the eye. Arrows indicate the maximum points. (c) Static dc magnetic field $H_{\mathrm{dc}}$ needed to achieve the maximum ME response as a function of $N$. The solid line shows a linear fit. (d) Normalized ME voltage coefficient (ratio of the ME coefficient at a particular $\delta$ and the maximum ME coefficient) as a function of magnetic-electric layer thickness ratio $\delta$ for the present study and theoretical predictions from Ref. 12, as indicated.

The increase in the ME coefficients can be attributed to an increase in the effective strain on the PZT fibers. For a structure with $N=2$ on both sides of PZT fibers, the total metglas-PZT thickness ratio $\delta$ is 0.5 . Expansion in the metglas with magnetic field results in a maximum expansion of the PZT layers close to the interface, which relaxes with distance in the PZT layers away from the interface. With an increase in the number of metglas layers to $N=6$, the thickness ratio increases correspondingly by three times. This, in turn, results in larger expansions in all the PZT layers (both close to and away from interface) giving rise to a maximum ME response for the 6 layered structure. However, as one increases the number of layers to $N>6$, the total laminate volume increases without any additional expansion in the PZT layers, resulting in an effective decrease in the ME coefficients. The small increase in the static field required to achieve the maximum ME response with increasing metglas thickness indicates a magnetic flux dilution in the PZT fibers. Clearly, our six layered structure with $\delta=1.5$ shows a much better ME response than the conventional two layered ones. Correspondingly, this offers significant improvements in the magnetic field sensitivity for the ME sensors. The difference in the $\delta$-values required to achieve the maximum ME response obtained from the present study and the predictions in Ref. 12 [as shown in Fig. 2(d)], presumably originates from the difference in the geometries and piezomagnetic responses for the structures.

Magnetic field sensitivity and output noise measurements were done using a home built low noise charge amplifier detection circuit of gain $1 \mathrm{~V} / \mathrm{pC}$ over the frequency range $0.16-10 \mathrm{~Hz}$. The structure was placed in an aluminum 

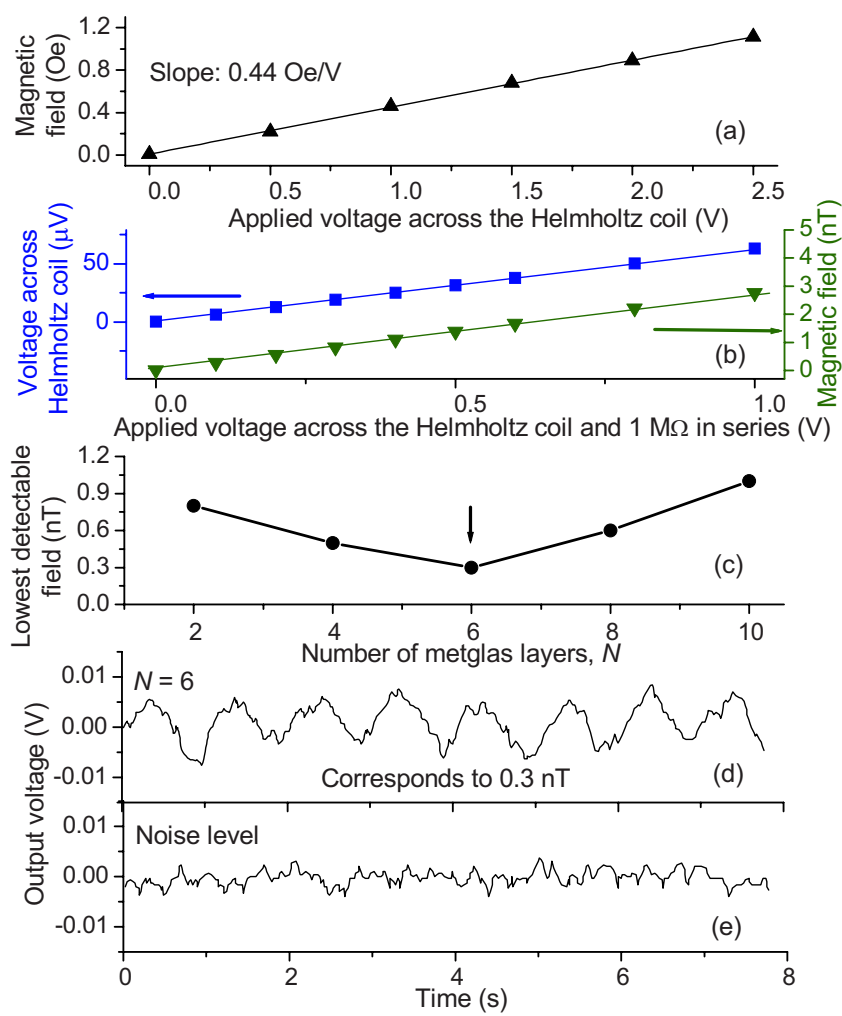

FIG. 3. (Color online) (a) Calibration results for ac magnetic field as a function of voltage applied directly to the Helmholtz coils. (b) Measured voltage across the coils (blue squares) and corresponding ac magnetic field (green triangles) as a function of applied voltage across the coils and $1 \mathrm{M} \Omega$ resistance in series. The solid lines indicate linear fits. (c) Lowest detectable magnetic field for the PZT fiber-metglas laminate composites as a function of the number of metglas layers $N$ on either side of PZT at $1 \mathrm{~Hz}$ for constant SNR $>2$. The arrow indicates the highest sensitivity point. Representative output (d) voltage waveform for a laminate with $N=6$ and (e) noise level for the low noise charge amplifier as a function of real time. The corresponding magnetic field sensitivity for $N=6$ is also labeled in (d).

box and was positioned at the center of a pair of Helmholtz coils. A $1 \mathrm{M} \Omega$ resistance was connected in series with the $80 \Omega$ coils and small ac voltages in the range of $0.1-0.3 \mathrm{~V}$ at a frequency of $1 \mathrm{~Hz}$ were applied from a lock-in amplifier to obtain the ac magnetic field $H_{\mathrm{ac}}$. Permanent magnets were used for the application of $H_{\mathrm{dc}}$ along the longitudinal axis of the structure. The dc field for each structure was optimized following the data given in Fig. 2(c) to achieve the maximum $\mathrm{ME}$ response as well as the highest sensitivity. The structure, box, permanent magnets, coils, and detection circuit were then kept inside a mu-metal can to prevent any electromagnetic interference that might disrupt the detection of the magnetic fields. The output voltage and the noise level for different structures with various metglas layers were measured with an oscilloscope. The noise amplitude of our charge amplifier detection circuit was about $0.006 \mathrm{~V}$. The applied $H_{\mathrm{ac}}$ was varied to keep the peak-to-peak output voltage constant at about $0.015 \mathrm{~V}$ (to maintain a signal to noise ratio SNR $>2$ ). This allowed us to compare the field sensitivities measured with a constant SNR for different structures.

Figure 3 shows the magnetic field calibration and sensitivity results. Part (a) shows the ac magnetic field as a function of the voltage applied directly across the Helmholtz coils. The solid line is a linear fit with a slope of $0.44 \mathrm{Oe} / \mathrm{V}$. Part (b) shows the actual voltage across the coils as a function of voltage applied across the coils and a $1 \mathrm{M} \Omega$ resistance in series (blue squares), and the estimated magnetic fields (green triangles) using the calibration data shown in (a). One sees that the actual voltage and the magnetic field vary linearly with applied voltages (shown by solid lines). Moreover, it can be seen that voltages in the $0.1-0.3 \mathrm{~V}$ range applied across the coils and resistor generate ac magnetic fields $H_{\mathrm{ac}}$ in the 0.3-1 nT at the center of the coil. Part (c) shows the lowest detectable magnetic fields for the laminate structures as a function of the number of metglas layers $N$. One can see that the structure with 2 metglas layers can detect a magnetic field of $0.8 \mathrm{nT}$ with a SNR $>2$. With increasing $N$, the magnetic field sensitivity increases almost linearly with $N$ up to 6 layers. The structure with $N=6$ is capable to detect a magnetic field as small as $0.3 \mathrm{nT}$ (with a SNR > 2). This is a 2.7 times increase in sensitivity relative to the two layered structure. This increase in sensitivity is a direct consequence of the increase in the $\mathrm{ME}$ voltage and charge coefficients that results from an increase in the effective strain on the PZT fibers with increasing metglas thickness. Parts (d) and (e) show representative output waveform as a function of real time for a structure with $N=6$ and the voltage noise spectrum in the time domain from our low noise charge amplifier, respectively. One can see that the peak-to-peak output voltage is much higher than the noise level with a $\mathrm{SNR}>2$.

In summary, an optimization of the metglas layer thickness has been performed to achieve the best possible ME response and magnetic field sensitivity for metglas-PZT fiber-metglas sandwiched heterostructures. Such structures hold considerable promise for low cost, modest size, energy efficient, room temperature sensor applications. It has been found that a structure with six metglas layers on both sides of the PZT layer had 1.5-1.75 times higher ME voltage coefficients, and 2.7 times higher magnetic field sensitivities than the two layered ones.

We acknowledge the support from the Office of Naval Research (Grant No. N0094-04-10492).

${ }^{1}$ M. Fiebig, J. Phys. D 38, R123 (2005).

${ }^{2}$ R. Ramesh and N. A. Spaldin, Nature Mater. 6, 21 (2007).

${ }^{3}$ C.-W. Nan, M. I. Bichurin, S. Dong, D. Viehland, and G. Srinivasan, J. Appl. Phys. 103, 031101 (2008).

${ }^{4}$ J. Das, Y. Song, N. Mo, P. Krivosik, and C. E. Patton, Adv. Mater. 21, 2045 (2009).

${ }^{5}$ G. Srinivasan, E. T. Rasmussen, J. Gallegos, R. Srinivasan, Y. I. Bokhan, and V. M. Laletin, Phys. Rev. B 64, 214408 (2001).

${ }^{6}$ L. Yan, Z. Xing, Z. Wang, T. Wang, G. Lei, J. F. Li, and D. Viehland, Appl. Phys. Lett. 94, 192902 (2009).

${ }^{7}$ Z. Xing, J. F. Li, and D. Viehland, Appl. Phys. Lett. 91, 182902 (2007).

${ }^{8}$ S. X. Dong, J. F. Li, and D. Viehland, Appl. Phys. Lett. 83, 2265 (2003).

${ }^{9}$ S. X. Dong, J. F. Li, D. Viehland, J. R. Cheng, and R. Cross, Appl. Phys. Lett. 85, 3534 (2004).

${ }^{10}$ J. Zhai, Z. Xing, S. X. Dong, J. F. Li, and D. Viehland, J. Am. Ceram. Soc. 91, 351 (2008).

${ }^{11}$ S. X. Dong, J. Zhai, J. F. Li, and D. Viehland, Appl. Phys. Lett. 89, 252904 (2006).

${ }^{12}$ D. A. Filippov, G. Srinivasan, and A. Gupta, J. Phys.: Condens. Matter 20, 425206 (2008). 